\title{
Prevalence of rat and mouse sensitization in Asian patients with respiratory allergy
}

\author{
Panipak Temboonnark, ${ }^{1}$ Mongkhon Sompornrattanaphan, ${ }^{2}$ Witchaya Srisuwatchari, ${ }^{1}$ Punchama Pacharn, ${ }^{1}$ \\ Orathai Jirapongsananuruk, ${ }^{1}$ Nualanong Visitsunthorn ${ }^{1}$
}

\begin{abstract}
Background: Skin prick test (SPT) is useful in identifying rat and mouse sensitization.

Objective: To determine the prevalence of rat and mouse sensitization by using local and commercial allergen extracts.

Methods: Patients with allergic rhinitis or asthma were recruited. SPT of local and commercial rat and mouse allergen extracts were performed. The level of rat and mouse specific IgE (sIgE) was quantified in all patients with positive SPT and randomized patients with negative SPT.
\end{abstract}

Results: Two hundred and thirty patients, 108 male (47\%) and median age 14 years (3.2-63.5 years), were enrolled. Rat sensitization by SPT was $11.7 \%$ and mouse sensitization was $17.8 \%$. SPT result to local rat and commercial rat allergen extracts were moderately correlated $\left(r_{s}=0.51, p<0.001\right)$, while SPT result to local mouse and commercial mouse allergen extracts showed low correlation $\left(r_{s}=0.38, p<0.001\right)$. The concordance of SPT results between local rat and commercial rat allergen extracts was $90.4 \%$. Concordance between the local mouse and commercial mouse allergen extracts was $85.2 \%$. When compared with rat and mouse sIgE, the concordance of local rat, commercial rat and commercial mouse allergen extract were $>80 \%$ while that of local mouse was $54.4 \%$. No adverse effect was observed in SPT with any allergen or extract.

Conclusions: The prevalence of rat and mouse sensitization was low compared to the study in USA. SPT with local rat and mouse allergen extract was safe and showed good concordance with the SPT result of commercial allergen extracts and rat and mouse sIgE levels.

Key words: rat, mouse, sensitization, skin prick test, specific IgE, asthma, allergic rhinitis

\section{Citation:}

Temboonnark, P., Sompornrattanaphan, M., Srisuwatchari, W., Pacharn, P., Jirapongsananuruk, O., Visitsunthorn, N. (2022). Prevalence of rat and mouse sensitization in Asian patients with respiratory allergy. Asian Pac J Allergy Immunol, 40(4), 401-406. https://doi.org/10.12932/ap-231019-0667

\section{Affiliations:}

${ }^{1}$ Division of Allergy and Immunology, Department of Pediatrics, Faculty of Medicine Siriraj hospital, Mahidol University, Bangkok, Thailand

${ }^{2}$ Division of Allergy and Immunology, Department of Medicine, Faculty of Medicine Siriraj hospital, Mahidol University, Bangkok, Thailand

\author{
Corresponding author: \\ Nualanong Visitsunthorn \\ Division of Allergy and Immunology, Department of Pediatrics, \\ Faculty of Medicine Siriraj Hospital, Mahidol University, \\ 2 Wanglang Rd, Bangkoknoi, Bangkok 10700, Thailand \\ E-mail: nualanongv@yahoo.com

$$
\begin{array}{ll}
\text { Abbreviations/Acronyms: } \\
\text { SPT } & \text { skin prick test } \\
\text { sIgE } & \text { specific IgE } \\
\text { MWD } & \text { mean wheal diameter }
\end{array}
$$

\section{Introduction}

Asthma and related allergic disorders are one of the most common chronic diseases globally. The estimated prevalence of asthma and allergic rhinitis (AR) in the general population is $10-30 \%$ worldwide. ${ }^{1}$ In Thailand, the International Study of Asthma and Allergies in Childhood (ISAAC) Phase III study (2001) reported the prevalence of asthma among children aged 6-7 years was $15 \%$ and aged 13-14 years was $13.9 \%$. 
The prevalence of AR in children aged 6-7 years was $43.2 \%$ and aged $13-14$ years was $57.4 \% .^{2} \mathrm{~A}$ recent multicenter study in Bangkok reported the prevalence of AR in children aged 6-7 years was $15.0 \%$ and in 13-14 years was $17.5 \% .^{3}$ A study of wheeze prevalence in Bangkok estimated 14.6\% of 6-7 year old children and $12.5 \%$ of $13-14$ year old children age had wheeze. ${ }^{3}$

Previous studies have confirmed that sensitization to indoor allergens increases the risk of development of asthma and allergic rhinitis. ${ }^{4-6}$ Most common indoor allergens originate from animals, including house dust mite, cat, dog, mouse, rat and cockroach. Studies in urban environments have reported the prevalence of rat sensitization to be $19-21 \%{ }^{7,8}$ The prevalence of sensitization to rat allergens is directly related to exposure rates. ${ }^{9,10}$ Rat allergen sensitization and exposure have been associated with increased asthma morbidity among inner-city residents. ${ }^{8}$ The National Cooperative Inner-City Asthma Study (NCICAS) demonstrated that mouse allergens were detected in at least one room in $95 \%$ of the houses prevalent in inner-city households. ${ }^{11}$ The prevalence of mouse sensitization assessed by skin prick test (SPT) and/or specific IgE (sIgE) response was 18\% from NCICAS to $65.7 \%$ in other studies. ${ }^{12-14}$ Increasing levels of exposure to mice were associated with sensitization..$^{13}$ Sensitization to mouse and exposure to rodent environment were associated with increased asthma morbidity in children and adults. ${ }^{11,14-17}$

Identification of rat and mouse allergen sensitization in patients with respiratory allergy is helpful to design avoidance measures that can improve the patients' quality of life. SPT is a useful tool to evaluate aeroallergen sensitization. Unfortunately, rat and mouse allergen extracts are sometimes difficult to procure due to delayed importation of the commercial extracts. In addition, local rat and mouse species may be different than those available in commercial allergen extracts. Rat and mouse sensitization may also be measured by serum sIgE levels. However, this method is expensive, time consuming and invasive and is not suitable as a screening test. Therefore, the rat and mouse allergen extract preparation process was developed in the Siriraj Hospital, Mahidol University laboratory.

This study aimed to assess the prevalence of rat and mouse sensitization using local and commercial allergen extracts and also to compare the efficacy of local and commercial rat and mouse allergen extracts in skin prick testing patients with respiratory allergy.

\section{Methods \\ Study population}

From January 2018 to February 2019, patients aged $\geq 3$ years with physician diagnosed allergic rhinitis and/or asthma ${ }^{18,19}$ were recruited from the Department of Pediatrics and Department of Internal Medicine, Siriraj Hospital, Mahidol University, Bangkok, Thailand. Subjects with acute asthma exacerbation (Forced Expiratory Volume $1<70 \%$ ), severe atopic dermatitis (SCORAD > 50), pregnancy or chronic diseases such as autoimmune diseases, immune deficiency, or liver disease were excluded.
Antihistamine, systemic corticosteroids $\geq 20 \mathrm{mg} /$ day and topical corticosteroids were discontinued for at least 7 days before testing. The study was registered with Clinical Trials.gov NCT03645161. The Institutional Ethics Committee, Siriraj Hospital, Mahidol University, EC 789/2560 (EC2) approved the study. Written informed consent from parents or guardians and assent from children older than 7 years of age were obtained.

Clinical characteristics, allergic symptoms and environmental exposure were recorded. All subjects received SPT to local rat epithelial, commercial rat epithelial, local mouse epithelial and commercial mouse epithelial extracts. Subjects were also pricked with other common aeroallergens including Bermuda, Acacia, Johnson, Careless weed, Curvularia, Cladosporium, Penicillium, Aspergillus, Dermatophagoides pteronyssinus, Dermatophagoides farinae, cat, dog, American cockroach and German cockroach. sIgE to rat and mouse were performed in all patients with a positive SPT (mean wheal diameter, MWD, $\geq 3 \mathrm{~mm}$.) and in 30 controls from randomized SPT negative patients (MWD $<3 \mathrm{~mm}$.) (Figure 1)

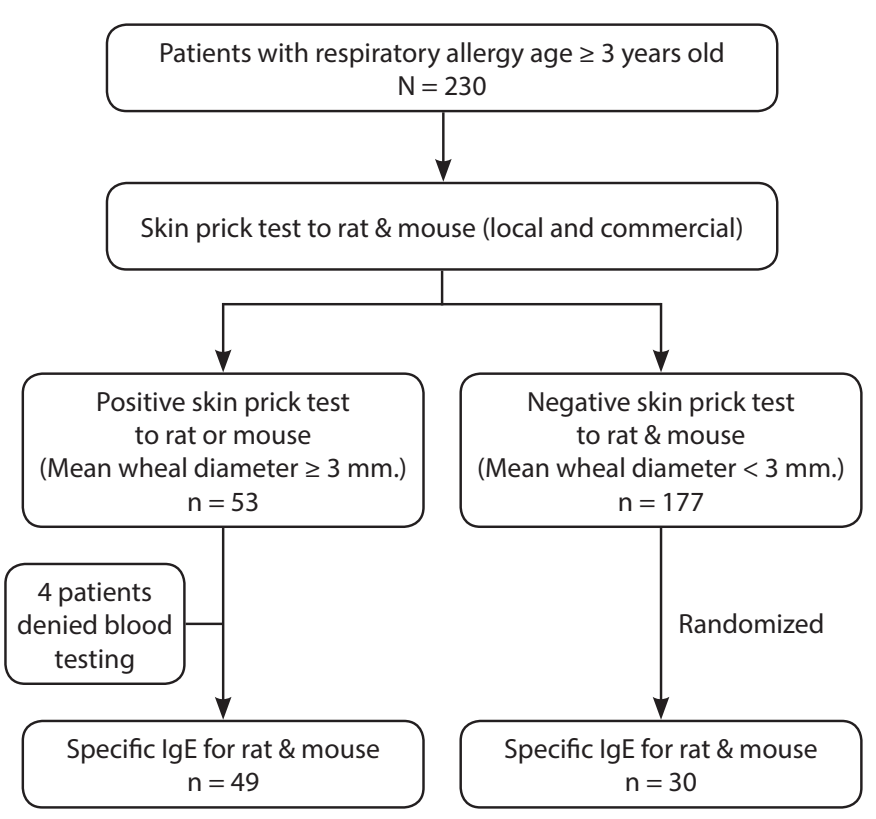

Figure 1. Study flow chart

\section{Rat and mouse local allergen extract preparation}

The extraction process developed by researchers at Siriraj Hospital, Mahidol University has been patented by the Thai regulatory authorities. The local rat (Rattus norvegicus) and mouse (Mus musculus) were raised in the Specified Pathogen Free system (SPF) by M-CLEA Bioresource Co., Ltd. Rat and mouse epithelia were added with phosphate buffered saline, $\mathrm{pH} 7.4$ (PBS), 0.1\% Tween-20 (1:20 w/v), and then incubated at $4^{\circ} \mathrm{C}$ for 30 minutes. The mixture was centrifuged at $8,000 \times \mathrm{g}, 4^{\circ} \mathrm{C}$ for 20 minutes. The supernatant was collected and mixed with glycerol $(1: 2)$ and filtrated through $0.2 \mu \mathrm{m}$ membrane filter. The filtrated solution was kept in a glass bottle at $4^{\circ} \mathrm{C}$. Protein concentration, sterility and single dose toxicity were tested before use. 
Commercial rat (Rattus norvegicus) epithelial allergen extract, 1:20 w/v (Greer Laboratories Lenoir, North Carolina) and mouse (Mus musculus) epithelial allergen extract, 1:20 W/V (ALK, Port Washington, New York) were used. Histamine dihydrochloride $(10 \mathrm{mg}$ base $/ \mathrm{ml})$ and sterile glycerinated saline were used as positive and negative controls, respectively. SPT of local commercial rat and mouse allergen extracts were performed with a blood lancet (Vitrex ${ }^{\circledast}$ steel, Vitrex Medical A/S, Herlev, Denmark) by an experienced technician in a room with full resuscitation equipment. SPT was performed on the patients' skin on the upper back in children and the volar surface of the forearm in adults. The presence of induced wheals and flares induced was recorded 10 minutes after positive control testing and 15 minutes after allergen extract testing. Mean Wheel Diameter (MWD) (the longest diameter plus the perpendicular diameter and divided by 2) was calculated. SPT was considered positive if the MWD was $\geq 3 \mathrm{~mm}$. larger than the SPT of negative controls.

\section{Specific IgE antibodies to rat and mouse allergen}

sIgE was measured in all patients who had a positive SPT result to rat or mouse allergen extract. sIgE was also measured in 30 randomized patients from a group of patients with negative SPT results to rat and mouse allergen extracts as negative controls. Randomization was performed using the program from www.randomization.com. sIgE to rat and mouse epithelia, serum and urine allergen were quantified using the ImmunoCap ${ }^{\circledR}$ (Phadia, Uppsala, Sweden). A level of $\operatorname{sIgE} \geq 0.35 \mathrm{kAU} / \mathrm{L}$ was considered positive.

\section{Statistical analysis}

Phipatanakul, et al. ${ }^{12}$ reported the prevalence of mouse sensitization in inner-city areas of the United State of America to be $18 \%$. We calculated a target sample size of 227 randomized participants to provide a 95\% confidence level and 5\% allowable error. The data were analyzed using SPSS software version 18 (SPSS Inc., Chicago, II., USA). Descriptive data are presented as the mean and standard deviation (SD) or median (range) for continuous data or number and percentage for categorical data. Agreement between SPT results of local and commercial allergen extracts was evaluated using kappa and intraclass correlation. ${ }^{20}$ Correlation coefficients between the different SPT allergen extract sources and between SPT and sIgE were evaluated using Spearman's rho correlation. ${ }^{21}$ The agreement between the SPT result of local and commercial allergen extracts and between SPT and sIgE were presented as percentage of concordance. Factors associated with rat or mouse sensitization were analyzed using the Mann-Whitney $U$ test. A $p$-value $<0.05$ was considered statistically significant. Potential predictors of rat and/or mouse sensitivity obtained at the time of enrollment included clinical characteristics, allergic symptoms, environmental exposure, and SPT results from other aeroallergens. Predictive power was estimated using univariate logistic regression analysis and then categorized to facilitate the calculation of odds ratios using multivariable logistic regression.

\section{Results}

Two hundred and thirty respiratory allergic patients with the median age of 14 years (range 3.2-63.5) were enrolled. Number (96.5\%) of study subjects had allergic rhinitis. One hundred and twenty-five (54.3\%) were children, 108 (47\%) were male, and more than half (56.5\%) resided in Bangkok, the capital city of Thailand. There were no significant differences in any demographic variables between patients with positive or negative SPT results. (Table 1) From 230 patients, $15(6.5 \%)$ were positive for local rat epithelial extract SPT and 17 (7.4\%) were positive for commercial rat epithelial extract. Unexpectedly, more patients had positive responses to commercial mouse epithelium (15.7\%) compared to local mouse epithelial extract (5.2\%). (Table 2) Number (6.5\%) of subjects had positive SPT to both rat and mouse allergen extracts. Fifteen of 27 (55\%) of patients who had positive SPT results to rat allergen extracts also had positive SPT results to mouse allergen extracts, while 15 of $41(36 \%)$ of patients who had positive SPT results to mouse allergen

Table 1. Baseline Patient Characteristics

\begin{tabular}{|c|c|c|c|}
\hline \multirow[b]{2}{*}{ Character } & \multicolumn{2}{|c|}{ Skin prick test $(\mathrm{SPT})$ to rat and/or mouse $(\mathrm{N}=230)$} & \multirow[b]{2}{*}{$p$-value } \\
\hline & $\begin{array}{l}\text { Positive SPT to rat or mouse } \\
\text { (local and commercial) } \\
(n=53)\end{array}$ & $\begin{array}{l}\text { Negative SPT to rat and mouse } \\
\text { (local and commercial) } \\
(\mathbf{n}=177)\end{array}$ & \\
\hline \multicolumn{4}{|l|}{ Age (year): } \\
\hline median (range) & $11.4(3.2-63.5)$ & $15.3(3.3-62.7)$ & 0.22 \\
\hline$<18(\mathrm{n}=125)$ & $8.2(3.2-13.5)$ & $8.8(3.3-17.4)$ & 0.52 \\
\hline$>18(\mathrm{n}=105)$ & $26.7(18.1-63.5)$ & $30.0(19.4-62.7)$ & 0.23 \\
\hline \multicolumn{4}{|l|}{ Gender } \\
\hline Male & $29(54.7 \%)$ & $79(44.6 \%)$ & 0.20 \\
\hline \multicolumn{4}{|l|}{ Urban versus Rural } \\
\hline Urban (Bangkok) & $33(62.3 \%)$ & $97(54.8 \%)$ & 0.34 \\
\hline Rural & $20(37.7 \%)$ & $80(45.2 \%)$ & \\
\hline
\end{tabular}


Table 1. (Continued)

\begin{tabular}{|c|c|c|c|}
\hline \multirow[b]{2}{*}{ Character } & \multicolumn{2}{|c|}{ Skin prick test $(\mathrm{SPT})$ to rat and/or mouse $(\mathrm{N}=\mathbf{2 3 0})$} & \multirow[b]{2}{*}{$p$-value } \\
\hline & $\begin{array}{l}\text { Positive SPT to rat or mouse } \\
\text { (local and commercial) } \\
\qquad(\mathbf{n}=53)\end{array}$ & $\begin{array}{l}\text { Negative SPT to rat and mouse } \\
\text { (local and commercial) } \\
\qquad(\mathrm{n}=177)\end{array}$ & \\
\hline \multicolumn{4}{|l|}{ Atopy } \\
\hline Atopic dermatitis & $7(13.2 \%)$ & $11(6.2 \%)$ & 0.15 \\
\hline Asthma & $8(15.1 \%)$ & $19(10.7 \%)$ & 0.39 \\
\hline Allergic rhinitis & $51(96.2 \%)$ & $171(96.6 \%)$ & 1.00 \\
\hline Food allergy & $11(20.8 \%)$ & $25(14.1 \%)$ & 0.24 \\
\hline \multicolumn{4}{|c|}{ Family incomes (Baht/month) } \\
\hline$\leq 25,000$ & $5(10.9 \%)$ & $19(11.6 \%)$ & 0.81 \\
\hline $25,001-50,000$ & $21(45.7 \%)$ & $62(37.8 \%)$ & \\
\hline $50,001-100,000$ & $15(32.6 \%)$ & $61(37.2 \%)$ & \\
\hline$>100,000$ & $5(10.9 \%)$ & $22(13.4 \%)$ & \\
\hline
\end{tabular}

Table 2. Patients with positive skin prick test to local or commercial mouse or rat allergen extracts $(N=230)$

\begin{tabular}{|l|l|c|}
\hline Rat & \multicolumn{1}{|c|}{\begin{tabular}{c}
\multicolumn{1}{|c|}{ Source of allergen extract used } \\
Local epithelium
\end{tabular}} & $\begin{array}{c}\text { Positive skin } \\
\text { prick test } \\
\text { No. (\%) }\end{array}$ \\
\hline & $\begin{array}{l}\text { Commercial epithelium } \\
\text { Local epithelium and commercial epithelium }\end{array}$ & $15(6.5)$ \\
\hline Mouse & $\begin{array}{l}\text { Local epithelium } \\
\text { Commercial epithelium }\end{array}$ & $27(11.7)$ \\
\hline $\begin{array}{l}\text { Rat and } \\
\text { mouse }\end{array}$ & $\begin{array}{l}\text { Local epithelium and commercial epithelium } \\
\text { Any kinds of allergen extracts }\end{array}$ & $76(15.7)$ \\
\hline
\end{tabular}

extracts had positive SPT results to rat allergen. Employing a combination of SPT positive results from local or commercial allergen extracts, the prevalence of rat and mouse sensitization were $11.7 \%$ and $17.8 \%$, respectively. (Figure 2) Local rat epithelium allergen extracts and commercial rat epithelium allergen extracts showed moderate correlation (correlation coefficient, $r_{s}=0.51, p<0.001$ ), while local and commercial mouse epithelium allergen extracts showed low correlation $\left(r_{s}=0.38, p<0.001\right)$. Fair agreement were found not only between local and commercial rat epithelium allergen extracts $($ kappa $=0.26)$ but also between local and commercial mouse epithelium

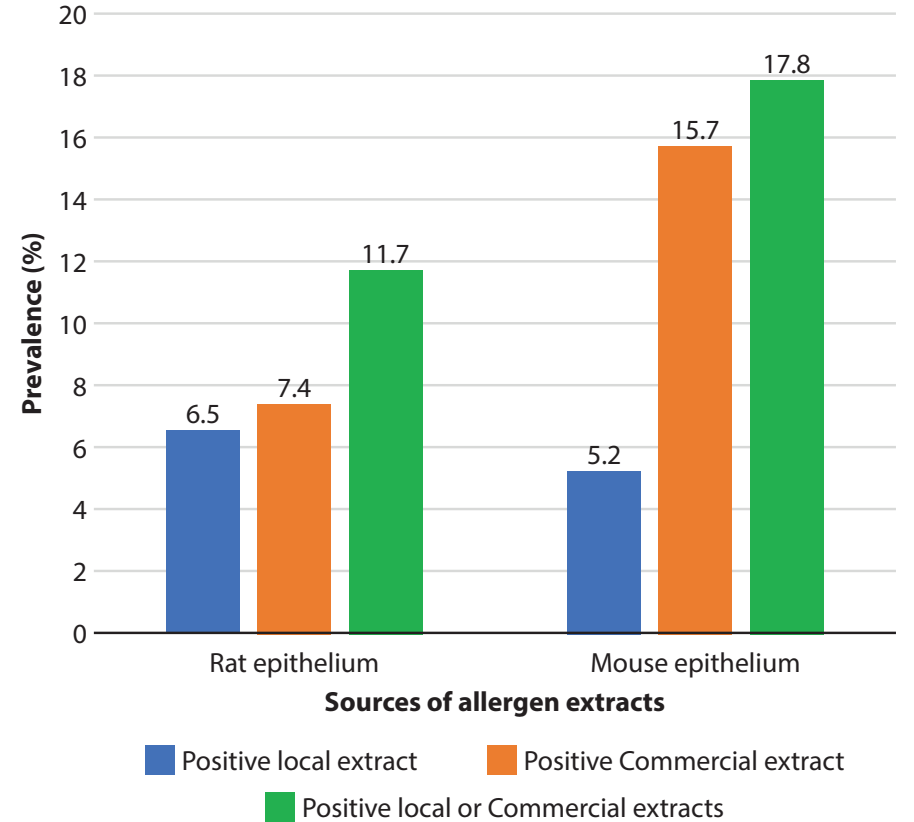

Figure 2. Prevalence of rat and mouse sensitization determined by skin prick testing

allergen extracts (kappa $=0.23$ ). The concordance of SPT results between local and commercial rat epithelium allergen extracts was $90.4 \%$ and between local and commercial mouse epithelium allergen extracts concordance was $85.2 \%$. (Table 3)

Serum sIgE was measured in 49 patients with positive SPT to rat or mouse allergen. The median sIgE level of rat was 0.01 $\mathrm{kUA} / \mathrm{L}(\max 82.5 \mathrm{kUA} / \mathrm{L})$ and mouse was $0.02 \mathrm{kUA} / \mathrm{L}(\max$ $48.2 \mathrm{kUA} / \mathrm{L})$. Rat sIgE was positive in 7 of 49 patients (14.3\%) and mouse sIgE was positive in 6 of 49 patients (12.2\%). In 30 randomized patients with negative SPT to rat and mouse, none had a positive sIgE to rat while one patient $(3.3 \%)$ had a positive sIgE to mouse allergen. 
Table 3. Concordance and agreement of skin prick test (SPT) results of local and commercial allergen extracts of rat and mouse $(\mathrm{N}=230)$

\begin{tabular}{|l|c|c|c|c|c|}
\multicolumn{1}{|c|}{ SPT } & $\begin{array}{c}\text { Positive local and } \\
\text { Positive commercial }\end{array}$ & $\begin{array}{c}\text { Positive local and } \\
\text { Negative commercial }\end{array}$ & $\begin{array}{c}\text { Negative local and } \\
\text { Positive commercial }\end{array}$ & $\begin{array}{c}\text { Negative local and } \\
\text { Negative commercial }\end{array}$ & $\begin{array}{c}\text { Concordance } \\
(\%)\end{array}$ \\
\hline Rat & 5 & 10 & 12 & 203 & 90.4 \\
Kouse & 7 & 5 & 29 & 189 & $0.26(p=0.09)$ \\
\hline
\end{tabular}

Table 4. Concordance and agreement of specific IgE (sIgE) and skin prick test (SPT) results of local and commercial rat and mouse allergen extracts $(n=79)$

\begin{tabular}{|c|c|c|c|c|c|c|}
\hline & $\begin{array}{l}\text { Positive SPT and } \\
\text { Positive sIgE }\end{array}$ & $\begin{array}{l}\text { Positive SPT and } \\
\text { Negative sIgE }\end{array}$ & $\begin{array}{l}\text { Negative SPT and } \\
\text { Positive sIgE }\end{array}$ & $\begin{array}{l}\text { Negative SPT and } \\
\text { Negative sIgE }\end{array}$ & $\begin{array}{c}\text { Concordance } \\
(\%)\end{array}$ & Kappa Coefficient \\
\hline \multicolumn{7}{|l|}{ Rat } \\
\hline Local & 5 & 10 & 2 & 62 & 84.8 & $0.46(p=0.14)$ \\
\hline Commercial & 5 & 9 & 2 & 63 & 86 & $0.38(p=0.14)$ \\
\hline \multicolumn{7}{|l|}{ Mouse } \\
\hline Local & 2 & 31 & 5 & 41 & 54.4 & $0.15(p=0.15)$ \\
\hline Commercial & 2 & 8 & 5 & 64 & 83.5 & $-0.54(p=0.07)$ \\
\hline
\end{tabular}

The correlation coefficient between local rat epithelium and rat sIgE was $r_{s}=0.20(p=0.08)$ and between commercial rat epithelium and rat $\operatorname{sigE}$ was $r_{s}=0.38$ $(p<0.001)$. The correlation coefficient between local mouse epithelium and mouse sIgE was $r_{s}=0.25(p=0.030)$ and between commercial mouse epithelium and mouse sIgE was $r_{s}=-0.04,(p=0.720)$.

The concordance between local rat allergen extract SPT results and rat sIgE level was $84.8 \%$, while commercial rat allergen extract concordance with rat sIgE level was $86.0 \%$. The concordance between local mouse allergen extract SPT results and mouse sIgE level was 54.4\%, while commercial mouse allergen extract concordance with mouse sIgE level was $83.5 \%$. Fair agreement was found between level of rat SIgE and MWDs from local rat epithelium allergen extract and MWDs from commercial rat epithelium allergen extract (kappa $=0.46$ and 0.38 , respectively). Slight agreement was found between level of mouse sIgE and MWDs from local mouse epithelium allergen extract (kappa $=0.15$ ) while poor agreement was found between level of mouse sIgE and MWDs from commercial mouse epithelium extract (kappa $=-0.54)$. (Table 4) No local or systemic adverse reactions related to any local or commercial allergen extracts were observed.

\section{Discussion}

We observed the prevalence of rat and mouse sensitization in children and adults with respiratory allergy (allergic rhinitis and/or asthma) to be $11.7 \%$ and $17.4 \%$, respectively. Previous studies among children with asthma reported 2-4 times higher prevalence of rat $(19-21 \%)^{7,8}$ and mouse sensitization (18-65.7\%). ${ }^{12-14}$ Important differences in these studies include the study location, the age of the studied population and the underlying allergic diseases of the patients.
Our study in Thailand included both children and adults while the other studies were performed in United States of America and included mostly children. Ninety six percent of our study subjects had allergic rhinitis, while the previous studies focused on patients with asthma. ${ }^{7,8,12-14}$

The prevalence of patients who had positive SPT results to the local rat epithelial extracts $(6.5 \%)$ was close to that of commercial rat epithelial extracts (7.4\%). Moderate correlation (correlation coefficient, $\mathrm{r}_{\mathrm{s}}=0.51, p<0.001$ ) and high concordance $(90.4 \%)$ were found between local and commercial rat allergen extracts. These results suggest that our local rat epithelial extract is suitable for screening of rat sensitization in Thailand.

In contrast, the percentage of patients with a positive SPT to mouse allergen extract was higher using commercial mouse epithelial extract (15.7\%) than with local extract (5.2\%). The correlation between local and commercial mouse allergen extracts was low $\left(\mathrm{r}_{\mathrm{s}}=0.38, p<0.001\right)$. This may be due to the difference in preparation methods, lack of standardization of local allergen extracts and differences of their allergenicity. We also found that the positivity of SPT was increased to $17.8 \%$ when we combined the results of local and commercial allergen extracts. However, we could not conclude that either extract was superior since the percentage of sensitization may not reliably indicate the presence of clinical allergy. To determine this, the gold standard respiratory provocation test for clinical sensitivity ${ }^{22}$ should be performed.

Rat and mouse allergens can be obtained from epithelium, serum and urine. Urine is believed to be the major source of rat and mouse allergenic protein ${ }^{23}$ because the major allergen of rat (Rat $n 1$ ) and mouse (Mus $\mathrm{m} \mathrm{1}$ ) is excreted in large amounts in the urine. ${ }^{24}$ However, the preparation of urine allergen extract is challenging. A pilot study was performed 
with local rat and mouse urine allergen extracts prepared by our researchers but this yielded sufficient extract to skin test only 70 patients. The low rates of positivity of local rat urine extract 8.6\% (6/70) and local mouse urine extract 7.1\% (5/70) prevented us from reliably estimating the correlation between SPT with local urine extracts and commercial extracts.

This is the first study to compare the prevalence of rat and mouse sensitization by SPT to local and commercial allergen extracts and sIgE in both pediatric and adult populations with respiratory allergy. This was also the first time that local rat and mouse antigen extracts were prepared for SPT in Thailand. Qualified personnel and standard laboratory were used in performing SPT and sIgE. We did not confirm rat or mouse allergy by respiratory provocation test so we report only the sensitization to rat and mouse allergens. Future studies should also include the respiratory provocation test in addition to SPT with rat and mouse urine allergen extracts. The preparation of local mouse epithelial allergen extracts also merits further study.

\section{Conclusion}

SPT with local rat and mouse allergen extract was safe and showed good concordance with the SPT result of commercial allergen extracts and rat and mouse sIgE levels.

\section{Acknowledgements}

The authors gratefully acknowledge Assoc. Prof. Dr. Nitat Sookrung for preparing local mouse and rat allergen extracts, Ms. Yuvaluk Khoprasert for the source and information of the rodent situation in Thailand, and Ms. Julaporn Pooliam for assistance with statistical analysis.

\section{Conflict of interest declaration}

All authors declare that they have no personal or professional conflicts of interest, and have not received any financial support from the companies that produce or distribute the drugs, devices, or materials described in this report.

\section{Funding disclosure}

This study was supported by a Chalermprakiat Grant from the Faculty of Medicine Siriraj Hospital, Mahidol University and National Science and Technology Development Agency (NSTDA) Chair Professor Grant (P-1450624) funded by the Crown Property Bureau.

\section{References}

1. Pawankar R, Canonica GW, Holgate ST, Lockey RF, Blaiss MS. WAO white book on allergy: update 2013. World Allergy Organization. 2013: $11-9$.

2. Trakultivakorn M, Sangsupawanich P, Vichyanond P. Time trends of the prevalence of asthma, rhinitis and eczema in Thai children-ISAAC (International Study of Asthma and Allergies in Childhood) Phase Three. J Asthma. 2007;44(8):609-11.

3. Chinratanapisit S, Suratannon N, Pacharn P, Sritipsukho P, Vichyanond P. Prevalence and severity of asthma, rhinoconjunctivitis and eczema in children from the Bangkok area: The Global Asthma Network (GAN) Phase I. Asian Pac J Allergy Immunol [Preprint]. 2018 [cited 2019 Oct 1]. Available from: http://apjai-journal.org/wp-content/ uploads/2018/10/AP-120618-0336.pdf
4. Lau S, Illi S, Sommerfeld C, Niggemann B, Bergmann R, von Mutius E, et al. Early exposure to house-dust mite and cat allergens and development of childhood asthma: a cohort study. Multicentre Allergy Study Group. Lancet. 2000;356(9239):1392-7.

5. Bjerg A, Ekerljung L, Eriksson J, Naslund J, Sjolander S, Ronmark E, et al. Increase in pollen sensitization in Swedish adults and protective effect of keeping animals in childhood. Clin Exp Allergy. 2016;46(10): 1328-36.

6. Sheehan WJ, Phipatanakul W. Indoor allergen exposure and asthma outcomes. Curr Opin Pediatr. 2016 ;28(6):772-7.

7. Perry T, Matsui E, Merriman B, Duong T, Eggleston P. The prevalence of rat allergen in inner-city homes and its relationship to sensitization and asthma morbidity. J Allergy Clin Immunol. 2003;112(2):346-52.

8. Kattan M, Mitchell H, Eggleston P, Gergen P, Crain E, Redline S, et al Characteristics of inner-city children with asthma: the National Cooperative Inner-City Asthma Study. Ped Pulmonol. 1997;24:253-62.

9. Hollander A, Heederik D, Doekes G. Respiratory allergy to rats: exposure-response relationships in laboratory animal workers. Am J Respir Crit Care Med. 1997;155(2):562-7.

10. Renström A, Karlsson AS, Malmberg P, Larsson PH, Van Hage-Hamsten M. Working with male rodents may increase risk of allergy to laboratory animals. Allergy. 2001;56(10):964-70.

11. Phipatanakul W, Eggleston PA, Wright EC, Wood RA. Mouse allergen. I. The prevalence of mouse allergen in inner-city homes. The National Cooperative Inner-City Asthma Study. J Allergy Clin Immunol. 2000; 106(6):1070-4.

12. Phipatanakul W, Eggleston PA, Wright EC, Wood RA, National Cooperative Inner-City Asthma Study. Mouse allergen. II. The relationship of mouse allergen exposure to mouse sensitization and asthma morbidity in inner-city children with asthma. J Allergy Clin Immunol. 2000;106(6):1075-80.

13. Fishbein AB, Lee TA, Cai M, Oh SS, Eng C, Hu D, et al. Sensitization to mouse and cockroach allergens and asthma morbidity in urban minority youth: Genes-environments and Admixture in Latino American (GALA-II) and Study of African-Americans, Asthma, Genes, and Environments (SAGE-II). Ann Allergy Asthma Immunol. 2016; 117(1):43-9.e1.

14. Ahluwalia SK, Peng RD, Breysse PN, Diette GB, Curtin-Brosnan J Aloe $\mathrm{C}$, et al. Mouse allergen is the major allergen of public health relevance in Baltimore City. J Allergy Clin Immunol. 2013;132(4): 830-5.e2.

15. Sedaghat AR, Matsui EC, Baxi SN, Bollinger ME, Miller R, Perzanowski $\mathrm{M}$, et al. Mouse sensitivity is an independent risk factor for rhinitis in children with asthma. J Allergy Clin Immunol Pract. 2016;4(1):82-8.e1.

16. Pongracic JA, Visness CM, Gruchalla RS, Evans R 3rd, Mitchell HE. Effect of mouse allergen and rodent environmental intervention on asthma in inner-city children. Ann Allergy Asthma Immunol. 2008; 101(1):35-41

17. Phipatanakul W, Litonjua AA, Platts-Mills TA, Naccara LM, Celedón JC, Abdulkerim $\mathrm{H}$, et al. Sensitization to mouse allergen and asthma and asthma morbidity among women in Boston. J Allergy Clin Immunol. 2007;120(4):954-6.

18. Brożek JL, Bousquet J, Agache I, Agarwal A, Bachert C, Bosnic-Anticevich S, et al. Allergic Rhinitis and its Impact on Asthma (ARIA) guidelines-2016 revision. J Allergy Clin Immunol. 2017;140(4): 950-8.

19. Global Strategy for Asthma Management and Prevention (updated 2019) [Internet]. [cited 2019 July]. Available from: https:/ginasthma.org/ gina-reports/.

20. Rosner B, Glynn RJ. Interval estimation for rank correlation coefficients based on the probit transformation with extension to measurement error correction of correlated ranked data. Stat Med. 2007;26(3):633-46.

21. Mukaka MM. Statistics corner: A guide to appropriate use of correlation coefficient in medical research. Malawi Med J. 2012;24(3):69-71.

22. Bernstein IL, Li JT, Bernstein DI, Hamilton R, Spector SL, Tan R, et al. Allergy diagnostic testing: an updated practice parameter. Ann Allergy Asthma Immunol. 2008;100(3 Suppl 3):S1-48.

23. Taylor AN, Longbottom JL, Pepys J. Respiratory allergy to urine proteins of rats and mice. Lancet. 1977;2(8043):847-9.

24. Matsui EC. Management of rodent exposure and allergy in the pediatric population. Curr Allergy Asthma Rep. 2013;13(6):681-6. 